Marina de Tommaso

Olimpia Difruscolo

Michele Sardaro

Giuseppe Libro

Carla Pecoraro

Claudia Serpino

Paolo Lamberti

Paolo Livrea

\section{Effects of remote cutaneous pain on trigeminal laser-evoked potentials in migraine patients}

Received: 12 March 2007

Accepted in revised form: 16 May 2007

Published online: 11 June 2007
M. de Tommaso ( $\square) \bullet$ O. Difruscolo • M. Sardaro • G. Libro • C. Pecoraro • C. Serpino • P. Lamberti • P. Livrea Neurophysiopathology of Chronic Pain Unit, Neurological and Psychiatric Sciences Department, University of Bari, Clinica Neurologica, Policlinico, Piazza Giulio Cesare 11, I-70124 Bari, Italy

e-mail: m.detommaso@neurol.uniba.it

Tel.: +39-080-5478565

Fax: +39-080-5478532

\author{
Abstract The present study aimed \\ to evaluate heat pain thresholds \\ and evoked potentials following \\ $\mathrm{CO}_{2}$ laser thermal stimulation \\ (laser-evoked potentials, LEPs), \\ during remote application of cap- \\ saicin, in migraine patients vs. \\ non-migraine healthy controls. \\ Twelve outpatients suffering from \\ migraine without aura were com- \\ pared with 10 healthy controls. \\ The LEPs were recorded by 6 \\ scalp electrodes, stimulating the \\ dorsum of the right hand and the \\ right supraorbital zone in basal \\ condition, during the application of \\ $3 \%$ capsaicin on the dorsum of the \\ left hand and after capsaicin \\ removal. In normal subjects, the \\ laser pain and the N2-P2 vertex
}

complex obtained by the hand and face stimulation were significantly reduced during remote capsaicin application, with respect to preand post-capsaicin conditions, while in migraine LEPs and laser pain were not significantly modified during remote painful stimulation. In migraine a defective brainstem inhibiting control may coexist with cognitive factors of focalised attention to facial pain, less sensitive to distraction by a second pain.

Keywords Migraine • Laser-evoked potentials $\cdot$ Diffuse noxious inhibiting control

\section{Introduction}

Recent evidence on migraine pathogenesis has emphasised the role of abnormal central pain processing in the initiation and maintenance of migraine $[1,2]$. It is likely that a network of cortical and subcortical structures with modulatory nociceptive and antinociceptive function becomes abnormally activated in a migraine attack or even between attacks [3].

The diffuse noxious inhibitory control (DNIC) allows that the activity of pain-transmitting neurons in the spinal dorsal horn and in trigeminal nuclei can be inhibited by noxious stimuli applied to body areas very remote from the excitatory fields of these neurons [4, 5]. Recently a reduced effect of remote noxious stimuli on pain threshold tested by electrocutaneous stimuli administered either to the forearm (extra-cranial site) or to the temple (cranial site) was observed in chronic tension-type headache, suggesting a deficient DNIC-like pain inhibitory mechanisms in this type of chronic headache [6]. Another recent study described the effect of heterotopic noxious conditioning stimulation by cold pressure test on the nociceptive flexion RIII reflex in 
chronic tension-type headache and migraine without aura patients, consisting in facilitation rather than inhibition of the reflex response in both types of headache [7].

The nociceptive flexion reflex is a reliable measure for pain circuits, but it is not able to test the processing of noxious inputs in the zones involved in headache. The laserevoked potentials (LEPs) obtained by the stimulation of the facial skin constitute a reliable neurophysiological method for the assessment of the trigeminal nociceptive pathways function [8]. In recent years we have performed some studies of LEPs in migraine. The most evident abnormalities consisted of a reduced habituation to repetitive stimuli [9], and a reduced inhibiting effect induced by distraction [2]; in addition, trigeminal LEPs did not appear inhibited during homotopical experimental pain [10]. In normal subjects, the LEPs obtained by limb and cervical dermatomal stimulation appeared suppressed by heterotopical noxious stimulation, for the effect of DNIC [11-13].

In a more recent study on healthy volunteers, trigeminal LEPs appeared reduced by heterotopical experimental tonic pain, either from muscle or from skin, for an effect mediated through the activation of segmental and suprasegmental inhibitory systems [14].

The aim of the present study was to test the effects of remote tonic pain induced by local application of capsaicin on trigeminal LEPs in migraine without aura patients.

\section{Methods}

\section{Subjects}

Twelve outpatients attending the Headache Centre of the Neurological Clinic of Bari University in Italy were enrolled in the study; they were affected by migraine without aura (MA) (cod. 1.1), according to the International Classification of Headache Disorders II criteria [15]. All subjects underwent a standardised interview, as well as a clinical neurological and psychiatric examination. Patients with general medical, neurological or psychiatric diseases, according to DSM-IV [16], were excluded from the study. No patients were taking psychoactive drugs or prophylactic treatment for headache at the time of the study. All selected headache sufferers were asked to attend the recording session (see below) in a pain-free state for at least $72 \mathrm{~h}$. The occurrence of the first migraine attack after the experimental task was defined in all cases, and patients who experienced headache in the $24 \mathrm{~h}$ following the recording session were excluded. The MA patients were 9 females and 3 males, aged 28-44 (mean age: $32.5 \pm 5.4$ ). The frequency of headache (days with headache/month) was computed in the last 2 months: it was $6.25 \pm 3.2$ days with headache/month. The mean interval since the last attack was $103 \pm 10.2 \mathrm{~h}$. Ten nonheadache healthy subjects, with no concomitant general, neuro- logical or psychiatric disease, selected among the technical staff of our department, served as controls: they were 2 males and 8

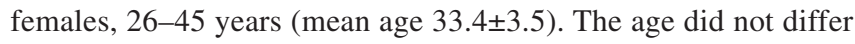
significantly across groups (Student's t-test: $0.21 \mathrm{~ns}$ ).

Laser-evoked potentials

LEPs were obtained using surface recording electrodes, placed at $\mathrm{Cz}$ and $\mathrm{Pz}$, referred to the nasion, and $\mathrm{T} 3$ and $\mathrm{T} 4$ positions, referred to Fz derivation, according to the 10-20 international system, by means of MICROMED EEG apparatus (Micromed Brain Quick, Mogliano, Veneto, Italy). The remaining electrode was positioned above the right eyebrow for electro-oculogram (EOG) recording. The ground electrode was at Fpz.

An off-line averaging was thus performed, after a manual deletion of the tracks containing ocular or muscular artefacts; in addition, an automatic artefact rejection algorithm excluded from the average all runs containing transient exceeding $\pm 65 \mathrm{mV}$ at any recording channel. A $0.3-70-\mathrm{Hz}$ bandpass filter, a pre-trigger analysis time of $100 \mathrm{~ms}$ and a post-trigger analysis time of 1000 ms with a bin width of $2 \mathrm{~ms}(500 \mathrm{~Hz}$ sampling rate) were employed.

LEP components were identified on the basis of their latency and polarity. They were labelled according to Valeriani et al. [18]. LEP amplitudes and latencies were measured from the baseline on the $\mathrm{CZ}$ and $\mathrm{T} 3$ derivations. Baseline was measured automatically by calculating the average signal on the whole sweep and subtracting it from the trace (ASA vers 4 by ANT software). In addition, the peak-to-peak amplitude was taken into consideration for the vertex biphasic LEP component (N2-P2).

During LEP recording, the subjects lay on a couch in a warm and semi-dark room; they were awake and relaxed, with eyes open. Both the subject and the experimenters wore protective goggles or glasses during data acquisition. Cutaneous heat stimuli were delivered by a $\mathrm{CO}_{2}$ laser (wavelength $10.6 \mathrm{Im}, 2 \mathrm{~mm}$ beam diameter, ELEN, Florence, Italy) on the dorsum of the right hand and the right supraorbital zone. We chose to limit the study to a right-sided stimulation in order to avoid a lengthy, uncomfortable procedure and because none of the selected migraineurs reported a higher attack prevalence on one side. The stimulation site was visualised by a He-Ne laser beam. The location of the impact on the skin was slightly shifted between two successive stimuli, to avoid the sensitisation of the nociceptors. $\mathrm{CO}_{2}$ laser stimuli were delivered at a fixed power of $7.5 \mathrm{~W}$ and duration of $25 \mathrm{~ms}$ [18], which was perceived by all patients and controls as a painful pinprick. We were careful to settle the laser power and duration at a suprathreshold level in all cases [18], using a 10-point visual analogue scale (VAS) in which ' 0 ' corresponds to no sensation, '4' to the Pain Threshold and ' 10 ' to intolerable pain. In all patients and controls the 25-ms duration and 7.5-W intensity laser stimuli were judged as a painful pinprick, with a VAS value $\geq 6$ in more than $50 \%$ of 20 stimuli on both the examined sites. 
Experimental procedure

Pain was induced by $3 \mathrm{ml}$ of $3 \%$ capsaicin in a cream base (Teofarma), which was applied topically by means of a cotton swab on the skin of the left-hand dorsum (radial territory), within an area of $6 \mathrm{~cm}^{2}$. We chose this method to study DNIC, though in analogous experiments other procedures were employed $[7,12,13]$, in order to give the minimum discomfort and side effects to patients, according to the indication of our local Ethic Committee (see below). The area of application was standardised by using an empty form, which was filled by capsaicin. Pain rating was performed by using a 100-point VAS, in which ' 0 ' corresponds to no pain and ' 100 ' to the worst pain one may conceive. Spontaneous pain induced by capsaicin was tested at intervals of $5 \mathrm{~min}$, and LEPs were performed when a clear sensation of pain, inducing in all subjects a VAS rating of at least 50, was induced. After each LEP recording, the subjects were asked to rate the pain induced by $\mathrm{CO} 2$ laser pulses (laser pain). LEPs were performed before capsaicin application, during capsaicin application and $1 \mathrm{~h}$ after capsaicin removal, delivering two series of 20 stimuli with a 9-11-s ISI and an inter-series interval of $5 \mathrm{~min}$ at the hand and supraorbital zones. The order of the three sessions was randomised between subjects. An average of the two series of LEPs obtained by the hand and face stimulation in the different conditions was performed in all cases.

All subjects selected for the study gave their informed consent prior to their inclusion, according to the 1964 Declaration of Helsinki: the study was approved by the Ethic Committee of the "Policlinico Consorziale" General Hospital, after the permission of the Neurological and Psychiatric Sciences Department of Bari University.

Statistical analysis

The pain ratings related to capsaicin application, the LEP features and laser pain ratings detected before capsaicin application were compared between groups by unpaired Student's $t$-test.

The LEP features and the VAS values related to laser pain were then compared within the groups by one-way ANOVA, considering the condition and the site of stimulation as factors and employing the Bonferroni as post hoc multiple comparison test among the three conditions.

LEP amplitudes and latencies and the laser pain ratings obtained during capsaicin recordings were expressed as percentages of the latencies, amplitudes and VAS values obtained in the pre-capsaicin condition, which were assumed as $100 \%$. After this normalisation, these parameters were designated as N1, N2, P2 latency and amplitude percentages and compared between groups by unpaired Student's $t$-test.

\section{Results}

Capsaicin induced a painful burning sensation in all cases: it started after 5 min and reached its peak after 30-35 min in patients and controls, when the recording session was started. The mean VAS values induced by capsaicin were $55.8 \pm 2.3$ in normal subjects and 57.4 \pm 3.2 in migraine patients (Student's $t$-test: 0.012; n.s.). It remained stable along the procedure. Capsaicin was removed in all cases after the recording session was completed: the pain induced on the dorsum of the left hand persisted up to 10-30 min in patients and controls.

\section{Basal pre-capsaicin condition}

The latencies and amplitudes of the N1, N2 and P2 components and the N2-P2 complex amplitude were similar in the two groups in the pre-capsaicin condition; the laser pain rating was also similar between groups (Table 1).

Effects of capsaicin application within groups

\section{Laser pain}

The rating of laser pain was significantly reduced in control subjects during capsaicin application with respect to preand post-capsaicin conditions at both the face and the hand levels (ANOVA with condition as factor: $F=3.21, p<0.05$; condition $\times$ site $F=0.21$, n.s.).

In migraine patients, the comparison between the three conditions was not significant at the hand and face levels (ANOVA with condition as factor: $F=1.86$, n.s.; condition $\times$ site, $F=1.98$, n.s.).

\section{LEP latencies}

In normal subjects, the latency of N1 was slightly prolonged, though not significantly, during capsaicin application (ANOVA with condition as factor: $F=2.38$, n.s.); the site $\times$ condition effect was not significant $(F=0.004$, n.s. $)$. The N2 latency was significantly prolonged during capsaicin application $(F=3.38, p<0.05)$ with respect to both preand post-capsaicin condition (Fig. 1). The latency prolongation was not different across the two sites of stimulation (condition $\times$ site: $F=1.44$, n.s.). The $\mathrm{P} 2$ latency was not significantly modified across the three conditions (ANOVA with condition as factor: $F=2.041$, n.s.; condition $\times$ site: $F=0.98$, n.s.).

In migraine subjects, the N1 latency was not modified during capsaicin application (ANOVA with condition as factor: $F=0.27$, n.s.; condition $\times$ site: $F=0.087$, n.s.). Similarly, the N2 and P2 latency did not vary significantly across the different conditions in the two sites of recording (N2: ANOVA with condition as factor: $F=0.39$, n.s.; condition $\times$ site: $F=0.5$, n.s. P2: condition: $F=0.84$, n.s.; condition $\times$ site: $F=0.9$, n.s.) (Fig. 1). 


\section{LEP amplitudes}

In control subjects, the $\mathrm{N} 1$ amplitude did not appear to be significantly reduced during capsaicin application at the face and hand levels (ANOVA with condition as factor: $F=1.26$, n.s.; condition $\times$ site: $F=0.03$, n.s.) (Figs. 1-3). Conversely, the $\mathrm{N} 2$ component showed a clear reduction in amplitude during capsaicin application at both levels of laser stimulation (condition as factor: $F=5.5, p<0.01$; condition $\times$ site: $F=0.35$, n.s.) (Figs. 1-3). Similarly, the P2 amplitude was also significantly reduced during capsaicin with respect to both the pre- and post-capsaicin conditions when the face and the hand were stimulated (condition as factor: $F=4.58, p<0.05$; condition $\times$ site: $F=0.027$, n.s.) (Figs. 1-3). In line with these findings, the N2-P2 complex evoked by both hand and supraorbital zone stimulation was also reduced for the effect of remote pain induced by capsaicin (condition as factor: $F=7.87, \quad p<0.001$; condition $\times$ site: $F=0.079$, n.s.) (Figs. $1-3$ ).

In migraine patients, the N1 amplitude was not modified by remote application of capsaicin at the hand and the face levels (condition: $F=0.6$, n.s.; condition $\times$ site: $F=0.78$, n.s.). The same applied for the $\mathrm{N} 2$ amplitude obtained by the hand and the face (condition: $F=0.68$, n.s.; condition $\times$ site: $F=0.087$, n.s.) (Figs. $1-3$ ). The P2 component was not reduced by remote pain when both the supraorbital zone and the hand were stimulated (condition: $F=0.72$, n.s.; condition $\times$ site: $F=0.64$, n.s.) (Figs. $1-3$ ). The $\mathrm{N} 2-\mathrm{P} 2$ complex remained similar across the different conditions at the two sites of laser stimulation (condition: $F=0.005$, n.s.; condition $\times$ site: $F=0.003$, n.s.) (Figs. 1-3).
Comparison between groups

\section{LEP latencies}

The N2 latency percentage was not significantly different between groups at either the hand or the face levels (Student's $t$-test: $1.16, \mathrm{n} . \mathrm{s}$.).

\section{Laser pain}

The comparison of the VAS percentage approached statistical significance between groups at the hand level (Student's $t$-test: 1.89 , n.s.) and was significant when the LEPs by face stimulation were considered (Student's $t$-test : 2.32, $p<0.05$ ).

\section{LEP amplitudes}

The N1 amplitude percentage was similar between groups (Student's $t$-test: hand: 0.12 , n.s.; face: 0.21, n.s.) (Fig. 4). The comparison of $\mathrm{N} 2$ amplitude percentage between groups was not significant at the hand ( $t$-test: $1.48, n . s$.) and the face levels ( $t$-test: 1.47, n.s.) (Fig. 4). Comparison of P2 percentage between groups approached statistical significance for the hand LEPs ( $t$-test: 1.98, $p=0.065)$, and was significant for the trigeminal LEPs $(t$-test: $2.46, p<0.05)$ (Fig. 4). The trigeminal N2-P2 complex percentage was also significantly different between groups ( $t$-test: $2.54, p<0.05)$. The comparison did not reach statistical significance when the N2-P2 obtained by the right hand was considered ( $t$-test: 1.96 , $p=0.063$ ) (Fig. 4).

Table 1 Laser evoked potentials latencies and amplitudes and laser pain rating expressed by 0-100 VAS for the right hand and the right supraorbital zone stimulation in pre-capsaicin condition in migraine without aura patients (MA) and non-migraine subjects (N). The unpaired Student's t test was not significant

\begin{tabular}{|c|c|c|c|c|c|c|c|c|c|}
\hline Hand & (msec) & $\begin{array}{r}\mathrm{N} 1 \\
(\mathrm{uV})\end{array}$ & $\begin{array}{r}\mathrm{N} 1 \\
(\mathrm{msec})\end{array}$ & $\begin{array}{r}\mathrm{N} 2 \\
(\mathrm{uV})\end{array}$ & $\begin{array}{r}\mathrm{N} 2 \\
(\mathrm{msec})\end{array}$ & $\begin{array}{r}\mathrm{P} 2 \\
(\mathrm{uV})\end{array}$ & $\begin{array}{r}\text { P2 } \\
(\mathrm{uV})\end{array}$ & $\mathrm{N} 2 \mathrm{P} 2$ & VAS \\
\hline \multirow[t]{3}{*}{ MA } & Mean & 164,37 & 9,34 & 207,37 & 14,18 & 346 & 16,3 & 29,89 & 48,91 \\
\hline & $\mathrm{N}$ & 12 & 12 & 12 & 12 & 12 & 12 & 12 & 12 \\
\hline & SD & 28,12 & 3,8 & 36,74 & 9,35 & 42,32 & 9,9 & 15,37 & 28,63 \\
\hline \multirow[t]{3}{*}{$\mathrm{N}$} & Mean & 158,6 & 9,27 & 217,1 & 14,34 & 337,8 & 17,28 & 31,6 & 32,45 \\
\hline & $\mathrm{N}$ & 10 & 10 & 10 & 10 & 10 & 10 & 10 & 10 \\
\hline & SD & 30,30 & 5,21 & 27,82 & 8,61 & 56,52 & 6,64 & 13,66 & 22,36 \\
\hline \multicolumn{10}{|l|}{ Face } \\
\hline \multirow[t]{3}{*}{ MA } & Mean & 136,33 & 11,21 & 161,83 & 18,23 & 287 & 13,89 & 32,2 & 49,96 \\
\hline & $\mathrm{N}$ & 12 & 12 & 12 & 12 & 12 & 12 & 12 & 12 \\
\hline & SD & 30 & 4,4 & 12,84 & 13,7 & 47,33 & 6,53 & 14,12 & 27,88 \\
\hline \multirow[t]{3}{*}{$\mathrm{N}$} & Mean & 130,2 & 7,83 & 160,5 & 19,38 & 321,3 & 18,36 & 37,92 & 37,9 \\
\hline & $\mathrm{N}$ & 10 & 10 & 10 & 10 & 10 & 10 & 10 & 10 \\
\hline & SD & 34,19 & 3,2 & 28,17 & 5,69 & 61,98 & 7,71 & 8 & 25,44 \\
\hline
\end{tabular}


Correlation between effects of capsaicin and clinical features in migraine patients

The correlation between the percentage of trigeminal P2 and N2-P2 amplitude in capsaicin vs. basal condition was not correlated with age (Pearson's correlation test: P2 percentage 0.544 , n.s.; N2-P2 percentage 0.25 , n.s.), age of illness (P2 percentage: 0.186, n.s.; N2-P2 percentage: 0.271, n.s.), frequency of headache (P2 percentage: 0.324 , n.s.; N2-P2 percentage: 0.289 , n.s.) or time elapsed from the last attack (P2 percentage: 0.021, n.s.; N2-P2 percentage: 0.121 , n.s.).

\section{Discussion}

In normal subjects, the remote painful stimulation induced by topical application of capsaicin significantly reduced the laser pain at the hand and face levels, with a correspondent inhibitory effect on the amplitude of the vertex N2-P2 component. In the post-capsaicin recording the laser pain and the LEPs returned to pre-capsaicin values, excluding that the reduction of VAS and LEPs amplitudes during the capsaicin application may be subtended by a long-term habituation effect. In previous studies, the effect of remote painful stimulation induced by hot and cold exposure reduced pain-evoked potentials and VAS score for the action of the DNIC [11-13]. In a study by Valeriani et al. [20] the topical application of $1 \mathrm{ml}$ of $3 \%$ capsaicin on the right hand failed to reduce laser pain and LEPs by the contralateral hand stimulation. In another study, where a more concentrated capsaicin solution was applied to the cheek, the LEPs obtained by the contralateral peri-oral region were inhibited for the effect of suprasegmental inhibitory system [14]. With respect to Valeriani et al. [20], in the pre-
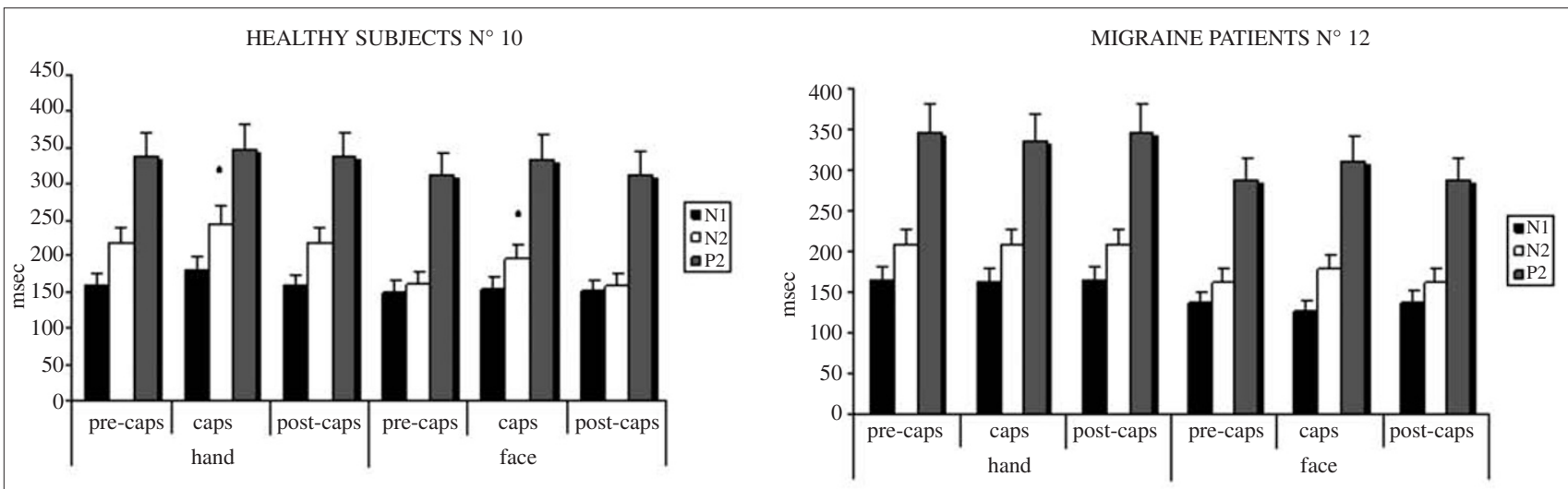

HEALTHY SUBJECTS N $=10$

MIGRAINE PATIENTS $N^{\circ} 12$
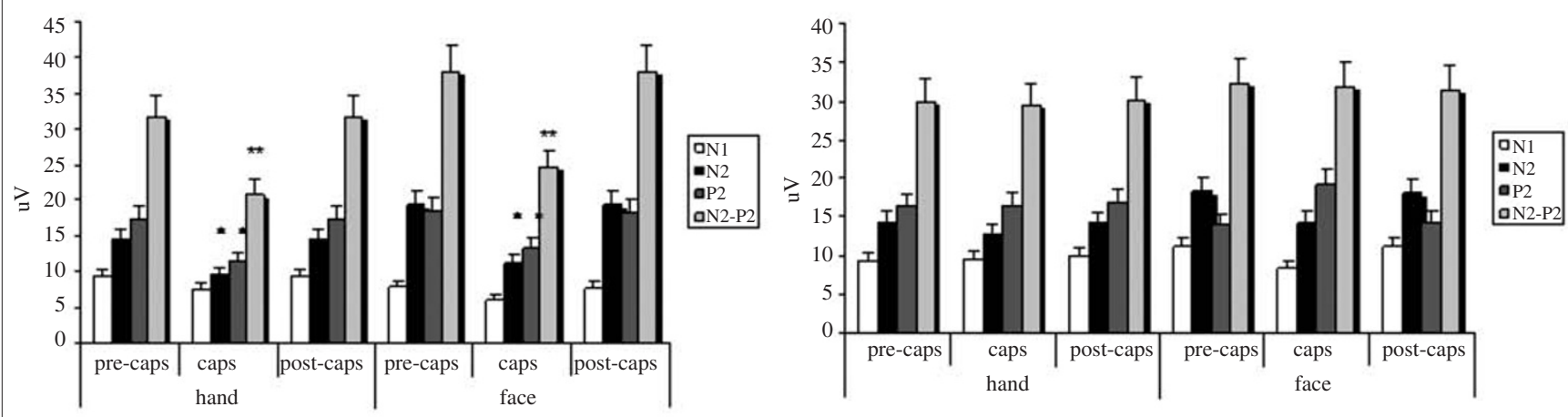

Fig. 1 Mean values and standard deviations of LEP latencies and amplitudes by right hand and supraorbital zone stimulation before, during and after capsaicin application on the dorsum of the left hand. Results of Bonferroni test are shown: capsaicin vs. pre- and post-capsaicin conditions. $* p<0.05, * * p<0.01$ 
sent study the same capsaicin concentration was applied in a major quantity on a larger zone of the hand, determining the reduction of LEPs evoked by the face and contralateral hand stimulation. The pain ratings induced by capsaicin were similar to those reported by Valeriani et al. [20] using a 0-100 VAS scale. In the study by Romaniello et al. [15], where a 0-10 VAS scale was employed, the subjective pain sensation induced by capsaicin seemed near the pain

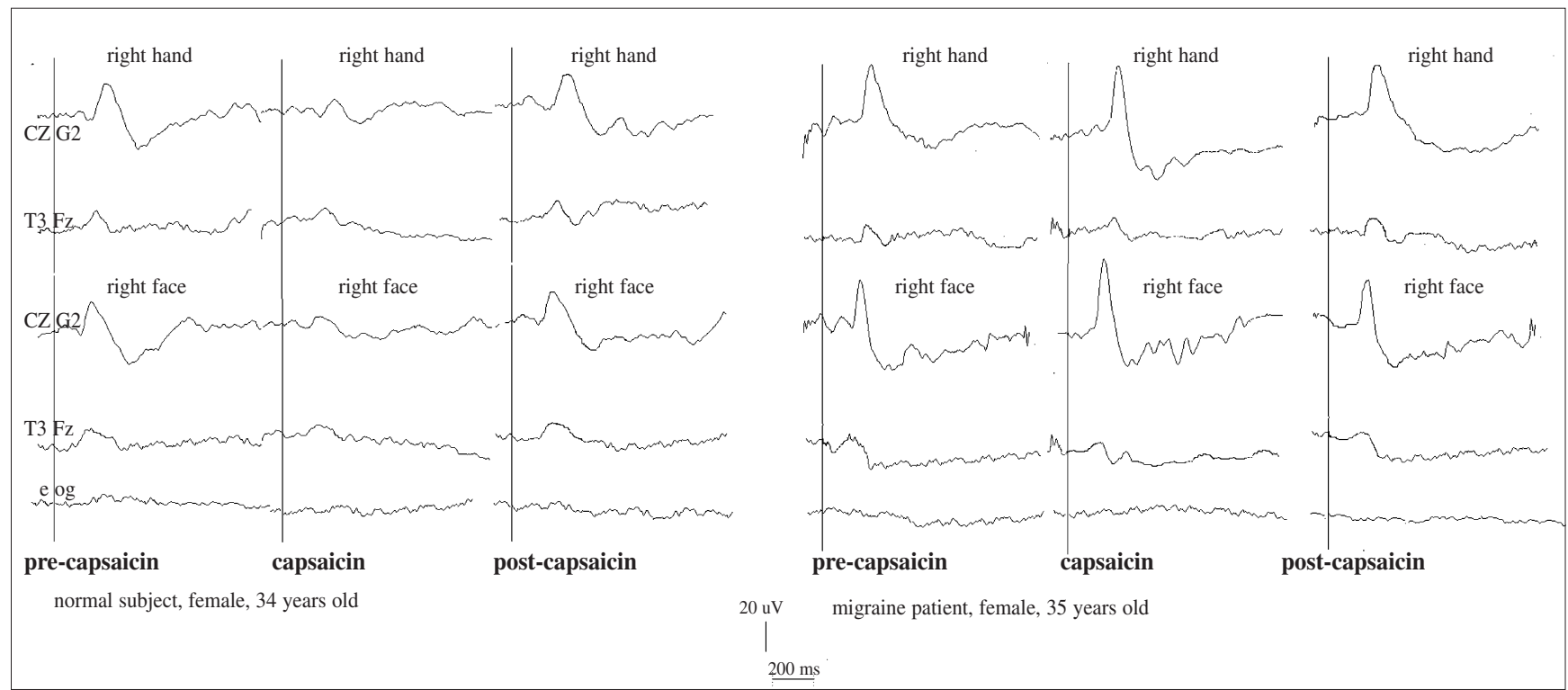

Fig. 2 An example of LEPs in a representative non-migraine healthy subject, female, 34 years old, and migraine without aura patients, female, 35 years old

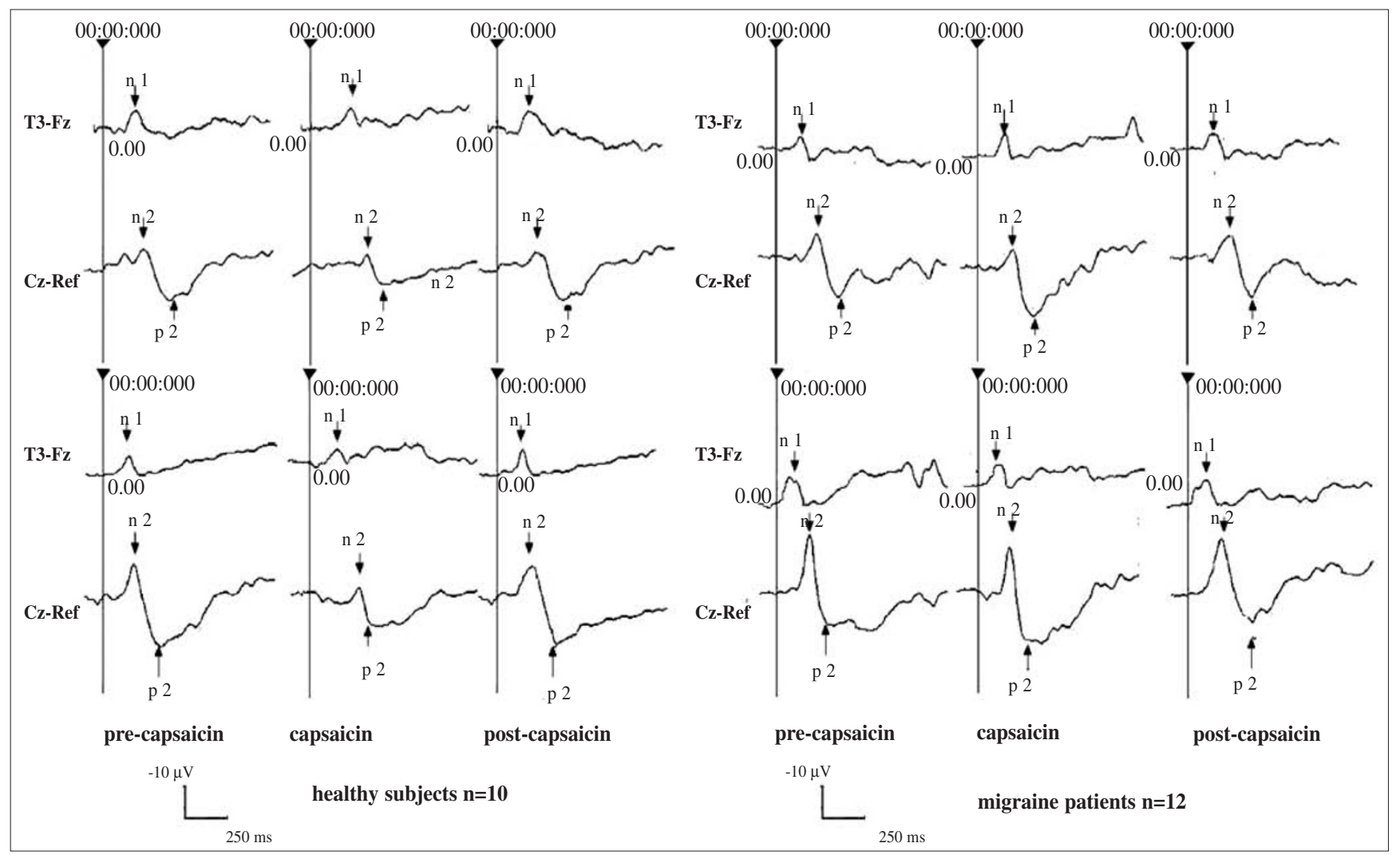

Fig. 3 Grand average of LEPs by the right hand and the face stimulation before, during and after capsaicin application on the dorsum of the left hand 
threshold levels. In the three studies the remote inhibitory effect seemed to be related to the concentration and extension of capsaicin application, despite similar pain ratings being observed, confirming the hypothesis that the subjective pain sensation may not always be considered an accurate measure for the state of sensory pathway [21]. The effect of DNIC was evident on the N2 and P2 amplitude and on the N2 latency, while a non-significant reduction of amplitude and prolongation of latency were induced on the N1 component at both the hand and trigeminal levels during the remote capsaicin application.

In previous studies, where only the vertex LEP components were evaluated, they appeared inhibited for the effect of DNIC [8, 11, 12], while the N1 component was not reduced by either homotopical or remote experimental pain [20]. According to the LEP dipolar model, the N1/P1 potential reflects the activity of the SII area contralateral to stimulation, while the vertex N2-P2 LEP component is mainly generated in the anterior cingulate gyrus [17].

The DNIC is considered to involve second-order convergent neurons in the dorsal horn, which give rise to the spinothalamic tract [22]; many of the projections from such convergent neurons reach the lateral thalamus, and then the parietal and opercular-insular cortices, where the N1 is generated [23]. It is difficult to conceive how a "massive" inhibitory effect taking place in the dorsal horn affected solely the projections to the cingulate, leaving intact those to the operculum, so we can conceive that the effect was present but not significant for technical limits, i.e., the relief of N1 amplitude solely on the T3 derivation. Though in this study topical capsaicin was used to evoke heterotopic pain, instead of heat, cold or electrical stimuli, it determined a clear pain sensation and an inhibitory effect on the N2-P2 complex, which may be attributed to a DNIC effect; anyway the lack of DNIC effect on N1 component in normal subjects needs to be confirmed, employing alternative methods to exert remote painful stimulation.

In view of the above remarks, an alternative hypothesis would be that the observed effect on LEPs and pain ratings was due to cognitive effects - i.e., distraction. Heterotopic pain should have strongly attracted attention in healthy subjects, and such "distractive" effects are sufficient to attenuate both pain ratings and LEPs to stimulation of another body site. As the effects of distraction are more conspicuous in vertex than in opercular LEPs [23] this might explain the dissociation observed here.

In migraine patients, the LEP features and the laser pain intensity in basal conditions were similar to those in nonmigraine subjects, according to previous studies [2, 9, 10]. During capsaicin application, the LEPs and laser pain were not modified at both the sites of examination, with respect to the pre- and post-capsaicin condition, despite a clear painful sensation being perceived on the left hand.
According to the above remarks, this phenomenon may be caused by two factors: a defect of DNIC and an altered cognitive processing of pain.

The defect of DNIC has been recently suggested in migraine and chronic tension-type headache on the basis of a facilitating instead of an inhibiting effect of remote pain on the RIII reflex response [7].

In the present study, the comparison with non-migraine subjects showed a significantly different behaviour of the P2 wave obtained by the face under capsaicin stimulation, with a consequent difference in amplitude modification of the N2-P2 vertex complex between groups. Accordingly, the correspondent VAS rate was significantly different between groups. In this study we observed a lack of statistical significance in the behaviour of P2 and N2-P2 amplitude at the hand level. Further evaluation in a larger series may confirm if the failure of the pain-inhibiting pain mechanism is a generalised phenomenon in migraine or alternatively if it is limited to the trigeminal level, but the present results outline the prevalence of a failure of DNIC control on the processing of the trigeminal noxious stimuli in migraine patients. The persistence of central sensitisation phenomena at the trigeminal level may not explain the present findings, considering that the trigeminal laser pain and evoked responses were found to be similar in migraine patients and controls in the basal precapsaicin condition. In addition, no correlation was found between the effects of remote pain on trigeminal LEPs and

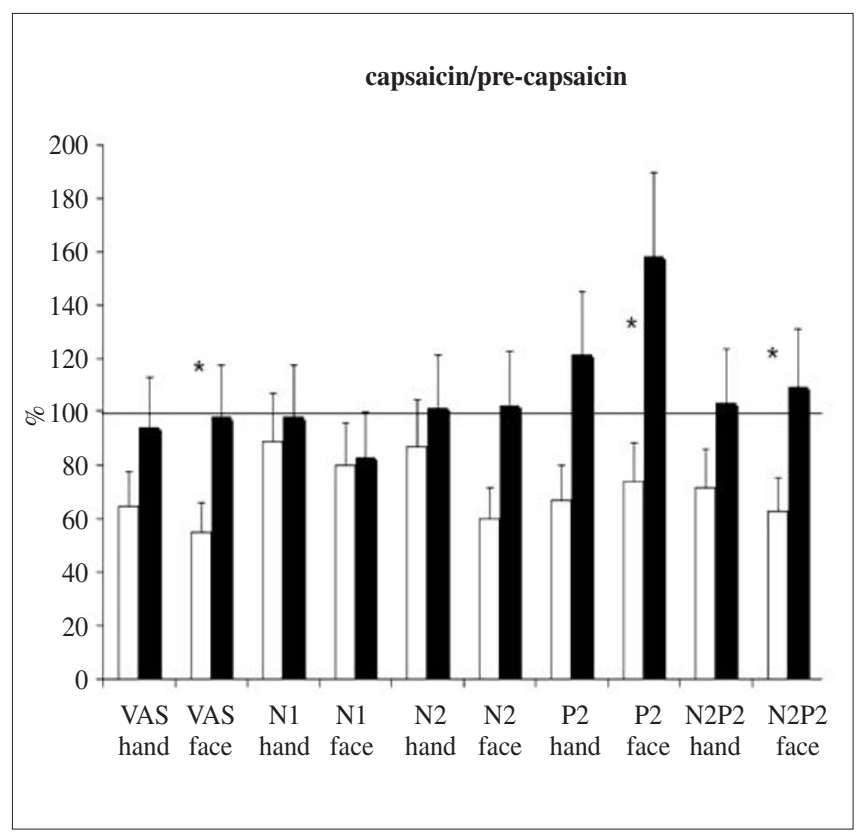

Fig. 4 Means and standard deviations of the percentage values between the VAS and the LEP amplitudes during capsaicin and before capsaicin application on the left hand. The results of unpaired Student's $t$-test are shown: $* p<0.05$ 
the time elapsed from the last attack.

Another explanation for the observed results is an altered cognitive processing of pain. In previous studies, migraine patients showed a defect in the attentive modulation of LEPs during an alternative cognitive task [4], so the direction of attention to the remote tonic pain induced by capsaicin may not be able to reduce the level of arousal and emotion toward the painful laser stimulation at the trigeminal level. In a further study, migraine patients did not show trigeminal N2-P2 complex reduction and changes of their cortical representation within the anterior cingulate cortex observed in non-migraine patients during homotopical painful stimulation concurrent with laser stimuli [11].

Though the failure of DNIC has been previously con- sidered a feature of chronic pain in general [5], in our patients there was no correlation between the reduced effect of remote pain on the LEPs and the frequency of headache, so it seemed a phenomenon linked with migraine per se rather than a sign of its severity.

In light of our results, it can be supposed that in migraine a defective brainstem inhibiting control and an altered attentive modulation of trigeminal pain may be taken into account to explain the extension and prolongation of central sensitisation phenomena occurring during an attack.

\section{Acknowledgements}

The study was supported by "Progetto per la Salvaguardia della

\section{References}

1. Burstein R, Yarnitsky D, Goor-Aryeh I, Ransil BJ, Baiwa ZH (2000)An association between migraine and cutaneous allodynia. Ann Neurol 47:614-624

2. Fields HL IHS Special Lecture (2001). Pain modulating networks in headache pathogenesis Cephalalgia 21:273

3. Welch KMA (2003) Contemporary concepts of migraine pathogenesis Neurology 61:S2-S8

4. de Tommaso M, Valeriani M, Guido M et al (2003) Abnormal brain processing of cutaneous pain in patients with chronic migraine. Pain 101:25-32

5. Dickenson AH, Le Bars D, Besson JM (1980) Diffuse noxious inhibitory controls (DNIC). Effects on trigeminal nucleus caudalis neurones in the rat Brain Res 200:293-305

6. Lautenbacher S, Rollman GB (1997) Possible deficiencies of pain modulation in fibromyalgia. Clin J Pain 13:189-196

7. Pielstickera A, Haagc G, Zaudig M, Lautenbacher S (2005) Impairment of pain inhibition in chronic tension-type headache. Pain 118:215-223

8. Sandrini G, Rossi P, Milanov I, Serrao M, Cecchini AP, Nappi G (2006) Abnormal modulatory influence of diffuse noxious inhibitory controls in migraine and chronic tension-type headache patients. Cephalalgia 26:782-789

9. Romaniello A, Iannetti GD, Truini A, Cruccu G (2003) Trigeminal responses to laser stimuli. Neurophysiol Clin 33:315-324
10. Valeriani M, de Tommaso M, Restuccia D et al (2003) Reduced habituation to experimental pain in migraine patients: a $\mathrm{CO}(2)$ laser evoked potential study. Pain 105:57-64

11. de Tommaso M, Losito L, Difruscolo O et al (2005) Capsaicin failed in suppressing cortical processing of $\mathrm{CO} 2$ laser pain in migraine patients. Neurosci Lett 384:150-155

12. Arendt-Nielsen L, Gotliebsen K (1992) Segmental inhibition of laser-evoked brain potentials by ipsi- and contralaterally applied cold pressor pain. Eur J Appl Physiol Occup Physiol 64:56-61

13. Kakigi R (1994) Diffuse noxious inhibitory control. Reappraisal by painrelated somatosensory evoked potentials following $\mathrm{CO} 2$ laser stimulation. J Neurol Sci 125:198-205

14. Watanabe S, Kakigi R, Hoshiyama M, Kitamura Y, Koyama S, Shimojo M (1996) Effects of noxious cooling of the skin on pain perception in man. J Neurol Sci 135:68-73

15. Romaniello A, Arendt-Nielsen L, Cruccu G, Svensson P (2002)

Modulation of trigeminal laser evoked potentials and laser silent periods by homotopical experimental pain. Pain 98:217-228

16. Headache Classification Subcommittee of the International Headache Society (2004) The International Classification of Headache Disorders. Cephalalgia 24[Suppl 1]:9-160
17. American Psychiatric Association. (1994) Diagnostic and statistical manual of mental disorders (DSM-IV), 4th ed. American Psychiatric Association Washington DC

18. Valeriani M, Rambaud L, Mauguie`re F (1996) Scalp topography and dipolar source modelling of potentials evoked by $\mathrm{CO} 2$ laser stimulation of the hand. Electroencephalog Clin Neurophysiol 100:343-353

19. Biehl R, Treede R-D, Bromm B (1984) Pain ratings of short radiant heat pulses. In: Bromm B, ed. Pain measurement in man Neurophysiological correlates of pain. Elsevier, Amsterdam pp 397-408

20. Valeriani M, Arendt-Nielsen L, Le Pera D et al (2003) Short-term plastic changes of the human nociceptive system following acute pain induced by capsaicin. Clin Neurophysiol 114:1879-1890

21. Treede R-D, Lorenz J, Baumgartner U (2003) Clinical usefulness of laserevoked potentials Neurophysiol Clin 33:303-314

22. Le Bars D, Dickenson AH, Besson JM (1979) Diffuse noxious inhibitory controls (DNIC). I. Effects on dorsal horn convergent neurons in the rat. Pain 6:283-305

23. Garcia-Larrea L, Peyron R, Laurent B, Mauguière F (1997) Association and dissociation between laser-evoked potentials and pain perception. NeuroReport 8:3785-3789 